\title{
ON THE COMMUTATOR AND FREDHOLMNESS OF ISOMETRIC PAIR
}

\author{
YIXIN YANG
}

Abstract. In this paper, we characterize the Fredholmness and compactness of commutators of the commuting isometric pair $W=\left(W_{0}, W_{1}\right)$ by using of their canonical model [3].

Mathematics subject classification (2010): Primary 47A45; Secondary 47A13.

Keywords and phrases: Bi-isometries, canonical model, commutator, Fredholmness.

\section{REFERENCES}

[1] C. Berger, L. Coburn And A. Lebow, Representation and index theory for $C^{*}$-algebras generated by commuting isometries, J. Funct. Analysis, 27 (1978), no. 1, 51-99.

[2] H. Bercovici, R. G. Douglas and C. Foias, On the classification of multi-isometries, Acta Sci. Math. (Szeged), 72 (2006), no. 3-4, 639-661.

[3] H. Bercovici, R. G. Douglas, And C. Foias, Canonical models for bi-isometries, in: Oper. Theory Adv. Appl., 218 (2012), 177-205.

[4] J. Conway, A course in functional analysis, Graduate Texts in Mathematics, vol. 96, 2nd edn., Springer, New York (1990).

[5] D. GASPAR AND P. GASPAR, Wold decompositions and the unitary model for bi-isometries, Integral Equations Operator Theory 49 (2004), no. 4, 419-433.

[6] K. Guo And P. WAng, Defect operators and Fredholmness for Toeplitz pairs with inner symbols, J. Operator Theory, 58 (2007), no. 2, 251-268.

[7] K. GuO And R. YANG, The core function of submodules over the bidisk, Indiana Univ. Math. J., 53 (2004), 205-222.

[8] W. HE, Y. QIN AND R. YANG, Numerical invariants for commuting isometric pairs, Indiana Univ. Math. J. (2015), 64, no. 1, 1-19.

[9] D. PopovicI, A Wold-type decomposition for commuting isometric pairs, Proc. Amer. Math. Soc. 132 (2004), no. 8, 2303-2314.

[10] B. Sz.-Nagy, C. Foias, H. Bercovici, And L. Kerchy, Harmonic analysis of operators on Hilbert space, Second Edition, Springer Verlag, New York, 2010.

[11] M. SŁocinski, On the Wold-type decomposition of a pair of commuting isometries, Ann. Polon. Math. 37 (1980), no. 3, 255-262.

[12] I. SucIu, On the semi-groups of isometries, Studia Math. 30 (1968), 101-110.

[13] R. YANG, Operator theory in the Hardy space over the bidisk 3, J. Funct. Anal., 186 (2001), 521-545.

[14] R. YANG, Hilbert-Schmidt submodules and issues of unitary equivalence, J. Oper. Theory. 53 (2005), 169-184.

[15] Y. YANG, Commutator of Hardy submodule over the bidisc, Houston J. Math., 41 (2015), no. 2, 611620. 